\title{
Oxidation of Aldoses With Bromine
}

\author{
Horace S. Isbell
}

\author{
(February 12, 1962)
}

\begin{abstract}
Rates of oxidation of aldoses with bromine have been reappraised and interpreted in the light of present concepts of conformation and reaction mechanism. It is suggested that differences in the rates of oxidation of the $\alpha$ and $\beta$ anomers are largely determined by differences in the free energy required by the reactants for passing from the ground state to the complex in the transition state. Structures for the aldoses in the ground states and in the transition states are postulated, and factors affecting the energy required for reaching the transition states from the ground states are discussed. The relative rates of oxidation are in accordance with the hypothesis that each of the aldoses in the ground state has the conformation predicted by Reeves, and, in the transition state, has a conformation in which the oxygen atom of the C1-hydroxyl group lies in the plane formed by the ring oxygen atom, C1, C2, and C5. Presumably, this conformation is stabilized by resonance involving the oxygen atom of the ring.

For aldoses having high stability in one chair conformation, the rates of oxidation of the anomers differ widely; in each instance, the anomer in which the C1-hydroxyl group is axial is oxidized more slowly than the anomer in which this group is equatorial. For aldoses having less stability in a chair conformation, the rates of oxidation of the anomers differ less widely, but, nevertheless, show a definite correlation with the angular position of the C1-hydroxyl group relative to the plane of the ring. For aldoses for which the stability in both chair conformations is so low that they probably exist in a variety of conformations, the rates of oxidation of the anomers show little difference and no particular correlation with the angular position of the C1-hydroxyl group.

The presence or absence of an oxygen atom in the ring is used to account for the large differences between the rates of bromine oxidation of the aldoses and those of derivatives of cyclohexanol. Differences in conformation in the transition state, associated with the presence or absence of this oxygen atom, likewise account for the fact that the relative rates of oxidation of the axial and equatorial isomers in the two classes of compound are reversed.

Because of uncertainty as to the anomeric configurations commonly assigned to some of the aldoses, the configurations of 22 aldoses were reappraised. Advantage was taken of the principle that the anomer preponderating in the equilibrium solution has trans hydroxyl groups at $\mathrm{C} 1$ and C2. Except for crystalline D-glycero-D-ido-heptose, the assignments of configuration based on this principle agree with the configurations generally accepted. Classificiation of crystalline D-glycero-D-ido-heptose as an $\alpha$-D-pyranose necessitates correction of earlier records in which this sugar was considered to be a $\beta$-D-pyranose.

In accordance with the author's earlier formulation, oxidation of the axial anomer is believed to take place by two courses: (1) direct oxidation and (2) conversion to the equatorial anomer by the anomerization reaction and the subsequent oxidation of this anomer. The relative importance of the two courses is not considered in this paper. It is pointed out, however, that the actual difference in the rates for the direct oxidation of the two anomers must be at least as great as that observed for the overall rates of oxidation.
\end{abstract}

\section{Discussion of Prior Work}

Utilization of bromine-oxidation measurements for study of the ring structure of aldoses began with the observation of Isbell and Hudson $[1,2]^{2}$ that, in slightly acid solution, pyranoses are oxidized to 1,5-lactones without cleavage of the ring. Isbell and Pigman $[3,4,5$ (pp. 435 to 456$)]$ showed that, for many aldoses, the $\alpha$ and $\beta$ anomers are oxidized at widely different rates, and Isbell [6] sought to classify the anomers of aldoses according to the angular position of the C1-hydroxyl group relative to the plane of a strainless, pyranoid ring. When those studies were made, the concept of conformation was just beginning to emerge. Nevertheless, it was concluded that the aldopyranoses exist in a definite conformation in which the $\alpha$ and $\beta$ positions are not symmetrically located with respect to the

1 Presented, in part, before the Division of Carbohydrate Chemistry at the 132d Meeting of the American Chemical Society, at New York, N.Y., on Septem ber $8,1957$.

2 Figures in brackets indicate the literature references at the end of this paper. plane of the ring, and that "a comparison of the reaction rates of alpha and beta pyranoses should provide information about the conformation of the pyranose ring"' (p. 524 of [6]). Subsequently, through the brilliant work of Reeves $[7,8]$, Hassel and Ottar [9], Beckett and co-workers [10], and others, factors that affect the conformation of the pyranoses have become known. Theoretical considerations as well as extensive experimental data have established that Isbell and Pigman's slowly oxidizable "alpha" anomers have a chair conformation with an axial C1-hydroxyl group and their rapidly oxidizable "beta" anomers have a chair conformation with an equatorial C1-hydroxyl group [11].

Kinetic studies have shown that oxidation by bromine is a complex process. Bunzel and Mathews [12] found that the reaction is rapid in neutral solution and slow in acid. Perlmutter-Hayman and Persky [13] recently confirmed the work of Bunzel and Mathews and presented evidence that the anionic form of D-glucose is oxidized much faster 
than D-glucose itself. Bunzel and Mathews also established that the rate of oxidation of pre-equilibrated D-glucose is first order with respect to the aldose and free bromine. Several investigators have advanced hypotheses to explain the difference in the rates of oxidation for the $\alpha$ and $\beta$ anomers. Isbell and Pigman [4] showed that oxidation of the separate anomers is complicated by the simultaneous anomerization reaction. ${ }^{3}$ In the oxidation of $\alpha-\mathrm{D}-$ glucose, formation of the more reactive $\beta$ anomer and oxidation thereof raises the overall rate of oxidation; in the oxidation of $\beta$-D-glucose, formation of the less reactive $\alpha$ anomer lowers the overall rate. Thus, the actual difference in the rates for the direct oxidation of the two anomers must be at least as great as the observed difference in the overall rates. Barker, Overend, and Rees [14] consider the values for the differences in the overall rates to be of little significance because of the anomerization of the slowly oxidizable anomers, which, they believe, takes place nearly completely before oxidation. Bentley [15] suggested that the higher activity of the anomers in which the C1-hydroxyl group is in an equatorial position arises from the release of strain in the ratedetermining step. He also noted that the ratios of rates of oxidation for the anomeric aldoses appear to be related to the conformational stability of the aldoses. The subject was discussed in an excellent review by Shafizadeh [16].

Various mechanisms have been proposed for the oxidation of aldoses with bromine. Isbell and Pigman [4] suggested rapid addition of free bromine to the ring oxygen atom, followed by slow elimination of hydrogen bromide at a rate depending on the stereomeric position of the C1-hydrogen atom. Lichten and Saxe $[17]$ postulated rapid addition of bromine to the anomeric oxygen atom; this is followed by elimination of the hydrogen atoms of $\mathrm{C} 1$ by base catalysis, and formation of two bromide ions. Friedberg and Kaplan [18] proposed elimination of the C1-hydrogen atom as a hydride ion by direct attack of bromine, a concept advanced by Kaplan [19] from study of the oxidation of ethanol and acetaldehyde. Bentley [15] suggested elimination of the C1-hydrogen atom as $\mathrm{Br}_{2} \mathrm{H}^{-}$from a bromine-containing intermediate. Isbell [20] suggested a bicyclic intermediate that decomposes intramolecularly with elimination of hydrogen bromide and a bromide ion (see p. 17 of [16]). Although the precise structure of the bromine-aldose complex in the transition state seems obscure, the structures given in figure 1 appear to account satisfactorily for many of the

\footnotetext{
${ }^{3}$ The relative rates of anomerization and oxidation depend on $p \mathrm{H}$, concentration of free bromine, and other factors. When the rate of anomerization is high in comparison with the rate of oxidation, there is little difference in the overall rates of oxidation for the anomers. Isbell and Pigman [4] represented the rate constant of oxidation for the anomers. Isbell and Pigman [4] represented the rate constant for the overall oxidation of $\alpha$-D-glucose as $a k_{\alpha}+k_{1}$, where $a$ is the concentration of
free bromine; $k_{\alpha}$, the rate constant for the direct oxidation of $\alpha$-D-glucose and $k_{1}$ free bromine; $k \alpha$, the rate constant for the direct oxidation of $\alpha$-D-glucose and $k_{1}$
the rate of conversion of the $\alpha$ anomer to the $\beta$ anomer and the oxidation thereof. It was assumed, as a rough approximation (when the bromine concentration is It was assumed, as a rough approximation (when the bromine concentration is
high), that $k_{1}$ corresponds to the rate of conversion of $\alpha$-D-glucose to $\beta$-D-glucose (calculated from the rate of mutarotation of $\alpha$-D-glucos s at an acidity and temperature comparable to the conditions prevalent during the measurements of oxidation). Under the conditions selected to minimize anomerization, it was estimated that about 80 percent of the overall oxidation of $\alpha$-D-glucose takes place directly, and 20 percent indirectly by conversion of $\alpha$-D-glucose to $\beta$-D-glucose and oxidation thereof. Evaluation of the relative importance of the direct oxidation and the indirect oxidation was not attempted for other aldoses because, in some instances, the mutarotation reaction involves several molecular spacies and the value of $k_{1}$ is highly uncertain (see p. 147 of [3]).
}
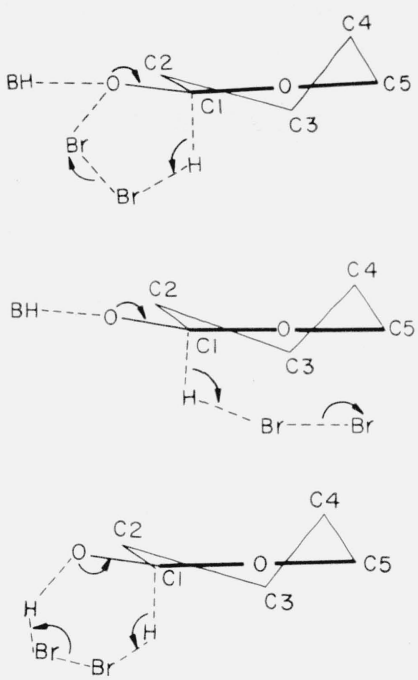

Figure 1. Hypothetical transition states.

(a) Intramolecular, base-catalyzed mechanism.

(c) Intramolecular, concerted mechanism.

experimental data. Structures (a) and (b) are symbolic of either a fast removal of a proton from the C1-hydroxyl group followed by a rate-determining hydride transfer from $\mathrm{C} 1$, or a partly concerted process in which the proton removal is not a completely separate step. Structure (b) is similar to one proposed by Swain, Wiles, and Bader [21] for the transition state in the oxidation of 2-propanol with bromine. Structure (c) is similar to an intermediate proposed by Barker, Overend, and Rees [22] for the oxidation of tert-butyl cyclohexanol, but differs in certain respects to be discussed in section 3 .

In a preliminary communication, Isbell [23] advanced the hypothesis that the relative rates of oxidation of aldoses depend on variation in the difference in free energy for the reactants in the ground state and in a hypothetical transition state in which the C1-hydroxyl group is restricted by resonance to the plane formed by the ring-oxygen atom, C1, C2, and C5. This concept will be developed in the next section of this paper.

\section{Rationalization of Reaction Rates in Terms of Free-Energy Differences}

In accordance with transition-state theory [24, 25, $26,27]$, the rate of reaction depends on the difference in free energy between the reactants in the ground state and an activated complex in the transition state. ${ }^{4}$ The difference in free energy depends solely on the nature of the two states and is independent of the manner in which the reaction proceeds. In the present study, the structure of the aldose was varied while other factors were held constant in so far as

${ }^{4}$ The free energy of activation $\Delta F^{ \pm}=-R T \ln K^{ \pm}$, where $K^{ \pm}$is the equilibriun constant for the aldose-bromine complex in the transition state. The free-ener change in passing from the ground state to the transition state involves chan in entropy as well as energy, and no attempt has been made to assess the relat importance of the two factors. 
possible. For this reason, the differences in rates of reaction can be assumed to arise for the most part from differences in free energy needed to convert the various aldoses to the structures characteristic of the transition states. ${ }^{4}$ The aldose in the ground state would have the conformation of lowest free-energy, probably the one selected by Reeves from consideration of instability factors [8]. In the activated intermediate in the transition state, the arrangement of the atoms of the carbohydrate portion would lie between that of the aldose and that of the lactone. Furthermore, stabilization by resonance would favor a transition state in which the anomeric oxygen atom lies in the same plane as $\mathrm{C} 1, \mathrm{C} 2, \mathrm{C} 5$, and the ringoxygen atom. Variation in the free energy involved in overcoming the intramolecular forces encountered in moving the atoms from the ground state to this transition state provides a basis for interpretation of the rates of reaction. Thus, with an aldose having an equatorial C1-hydroxyl group, a small movement at $\mathrm{C} 1$ would suffice to give the requisite arrangement of atoms for the transition state (see fig. 2); little energy would be required and the rate of reaction would be high. With an aldose having an axial C1hydroxyl group, formation of the transition state would require displacement of the anomeric oxygen atom from a position perpendicular to the pyranose ring to a position in the plane of the ring, in fact, a change in conformation. The free energy required for such a change depends on the structure of the entire molecule; and hence would be expected to differ for the various aldoses.

Table 1 gives a summary of the rates of oxidation (relative to the rate for $\alpha$-D-glucose) for the aldoses studied by Isbell and Pigman, as well as heretofore unpublished rate measurements for the oxidation of D-allose, D-altrose, and D-idose. The aldopyranoses are classified according to configuration and conformation; ${ }^{5}$ those of group 1 presumably have high stability in one of the chair conformations as predicted by Reeves, whereas those of groups 2 and 3 presumably have less stability.

In the ground state, the substances in group 1 have either the gluco-CA or galacto-CA conformation. ${ }^{6}$ The members of group 2 are considered to have one of the following conformations: manno-CA, talo-CA, or gulo-CA. The assignments are less certain than those of group 1. The members of group 3 do not appear to be especially stable in either chair conformation, and the conformations postulated are somewhat arbitrary.

The relative rates of oxidation for the axial anomers of the aldoses of group 1 average 1.4, whereas the relative rates for the equatorial anomers average

5 The system for indicating conformations is described in [28]. Briefly, a symbol (C, B, S, etc.) deseribing the type of ring is combined with a second symbol, $\mathrm{A}$ or $\mathrm{E}$. If the Cl-hydroxyl group of the $\alpha$ anomer is axial, the symbol $\mathrm{A}$ is used with the ring symbol; if this hydroxyl group is equatorial, the symbol $\mathrm{E}$ is used. The symbols are suffixed by a hyphen to the conventional name of the compound; for example, $\alpha$-D-glucopyranose-CA indicates that chair conformation of $\alpha$-D-glucopyranose that has an axial C1-hydroxyl group; $\beta$-D-glucopyranose-CA is the corresponding $\boldsymbol{\beta}$ anomer having the same chair conformation and therefore having an equatorial C1-hydroxyl group.

For reasons given in [29] and [30], D-xylose and L-arabinose are classified with D-glucose and D-galactose, respectively. D-Lyxose resembles both D-mannose and L-gulose, whereas D-ribose resembles both D-allose and L-talose. As regards chemical reactivity, there is, of course, no distinction between the $D$ and the $L$ series.

EQUATORIAL ANOMER

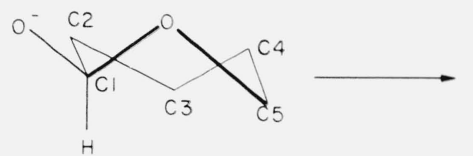

GROUND STATE

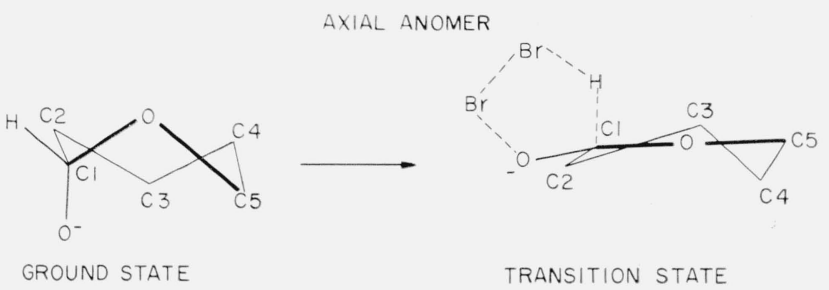

FIGURE 2. Ground states and transition states for the anions of the equatorial and axial anomers.

In the transition states, carbon atoms 1,2 , and 5 , as well as the oxygen atoms, tend to lie in the same plane.

49. The rates for the axial anomers of group 2 average 2.7 , whereas the rates for the equatorial anomers average 27. Thus, less energy is required for activation of the axial anomers of the aldoses of group 2 than for those of group 1, but more energy is required for activation of the equatorial anomers. In both groups, the modification having an equatorial C1hydroxyl group in the ground state is oxidized more rapidly than its anomer. Thus, the energy barrier in the oxidation is lower for the equatorial than for the axial anomers. The observations are in accord with the changes in free energy which might be anticipated in passing from the ground states to the transition states for the two anomers, in which a planar arrangement of $\mathrm{C} 1, \mathrm{C} 2, \mathrm{C} 5$, and the two oxygen atoms is stabilized by resonance.

With the axial anomers of the aldopyranoses of group 1, as depicted in figure 3 , rotation of $\mathrm{C} 1$ to form the transition state would bring the hydroxyl groups of $\mathrm{C} 1$ and $\mathrm{C} 2$ into alinement; because of strong repulsive forces, this eclipsing would require considerable energy. Turning of carbon atom 1 would also cause changes in the relative positions of the atoms at other carbon atoms. The sum of all the changes in free energy involved in reaching the transition state from the ground state would determine the rate of reaction.

Similar relationships can be noted for the aldoses of group 2. With the axial anomers of aldopyranoses having either the manno or the talo configuration, the C1-hydroxyl group would be eclipsed by the C2hydrogen atom (instead of by the C2-hydroxyl group, as in the aldoses of group 1). Undoubtedly, the change in conformation for these substances would require less energy than that required for the axial anomers of the aldoses of group 1, in accordance with the slightly higher rate of oxidation observed. With the axial anomers having the gulo configuration, rotation of $\mathrm{C} 1$ to form the transition state would bring the hydroxyl groups of $\mathrm{C} 1$ and $\mathrm{C} 2$ into alinement, 
TABLE 1. Relative rates of oxidation of aldoses with bromine a

\begin{tabular}{|c|c|c|c|c|c|c|c|}
\hline \multicolumn{4}{|c|}{ More reactive anomer } & \multicolumn{4}{|c|}{ Less reactive anomer } \\
\hline Aldose b & $\begin{array}{c}\text { Postulated } \\
\text { conformation } \\
\text { in ground } \\
\text { state }^{c}\end{array}$ & $\begin{array}{l}\text { Relative } \\
\text { rate of } \\
\text { reaction } d\end{array}$ & $\begin{array}{l}\text { Instability } \\
\text { factors for } \\
\text { conforma- } \\
\text { tion with } \\
\text { equatorial e } \\
\text { C1-OH }\end{array}$ & $\begin{array}{l}\text { Postulated } \\
\text { conformation } \\
\text { in ground } \\
\text { state }^{c}\end{array}$ & $\begin{array}{l}\text { Relative } \\
\text { rate of } \\
\text { reaction } \mathrm{d}\end{array}$ & $\begin{array}{c}\text { Instability } \\
\text { factors for } \\
\text { conforma- } \\
\text { tion with } \\
\text { equatorial } \\
\text { C1-OH }\end{array}$ & $\begin{array}{l}\text { Ratios of } \\
\text { overall rate } \\
\text { constants for } \\
\text { the anomers }\end{array}$ \\
\hline
\end{tabular}

Group 1

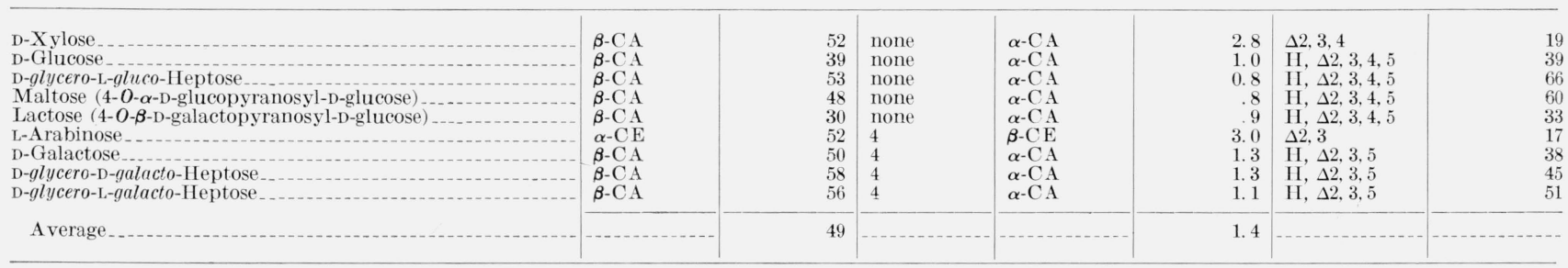

Group 2

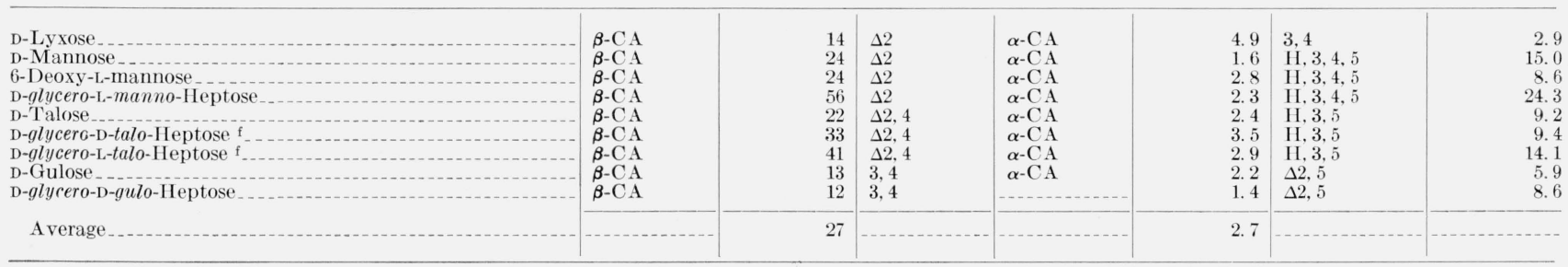

Group 3 (Oxidized at intermediate rates)

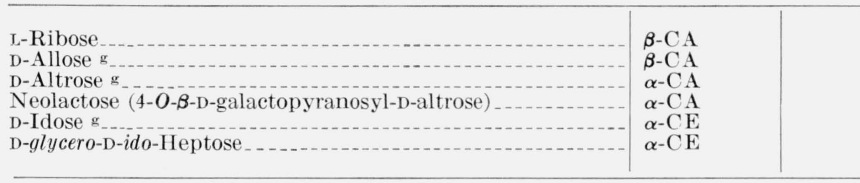

a Except where noted otherwise, the data are from the work of Isbell and Pigman reported on pages 436 and 455 of reference [5].

man reported on pages 436 and 455 of reference [5].

$\mathrm{b}$ Since the original publications
in nomenclature have been made:

n nomenclature have been made:

$\beta$-L-arabinose to $\alpha$-L-arabinose,

$\boldsymbol{\beta}$-L-ribose to $\alpha$-L-ribose,

$\mathrm{D}-\boldsymbol{\beta}$-galaheptose to D-glycero-L-gluco-heptose,

$\mathrm{D}-\alpha$-mannoheptose to D-glycero-D-galacto-heptose.

$\mathrm{D}-\boldsymbol{\beta}$-guloheptose to D-glycero-L-galacto-heptose,

$\mathrm{D}-\alpha$-galaheptose to D-glycero-L-manno-heptose,

D- $\alpha$-guloheptose to D-glycero-L-talo-heptose,

$\mathrm{D}-\boldsymbol{\beta}$-mannoheptose to D-glycero-D-talo-heptose,

D- $\alpha$-glucoheptose to D-glycero-D-gulo-heptose, and

D- $\boldsymbol{\beta}$-glucoheptose to D-glycero-D-ido-heptose.

\begin{tabular}{r|r|r|r|r|r}
6.1 & 3 & & & \\
5.0 & 3 & & & & \\
5.4 & 4,5 & & & & \\
5.6 & 4,5 & & & \\
6.5 & 5 & & &
\end{tabular}

c Postulated conformation based on the instability factors of Ree ves [8] expressed in the symbols of Isbell and Tipson [28].

d Based on the rate constant for $\alpha$-D-glucopyranose as unity.

e The instability factors are cited for the chair conformation having an equatorial Cl-OH group regardless of the actual conformation of the substance. For the more reactive anomers this conformation is presumably that of the ground state; for the less reactive anomers it is supposedly the less stable conformation. The numbers designate the individual carbon atoms bearing axial groups (other than hydrogen). H refers to the enhanced effect (Hassel and Ottar) of 2 axial groups on the same side of the ring, and $\Delta 2$ refers to a highly unstable arrangement of the carbon-oxygen bonds at carbon atoms 1 and 2 .

$f$ Values calculated from measurements reported in J. Research NBS 20, 97 (1938) RP1069.

g Unpublished measurements made by H. S. Isbell, R. Schaffer, and B. Y. Foley.

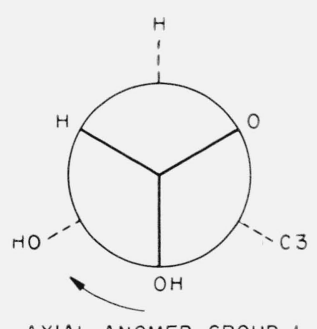

AXIAL ANOMER GROUP I

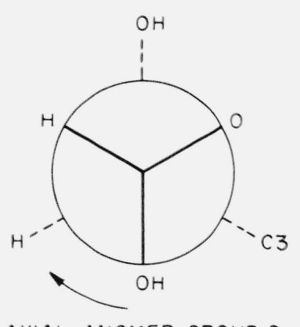

AXIAL ANOMER GROUP 2

Figure 3. Relative positions of atoms attached to carbon atoms 1 and 2.

(When $\mathrm{C} 1$ is turned on the C1-C2 axis, high-energy states arise from eclipsing of the groups of $\mathrm{C} 1$ and $\mathrm{C} 2$.)

as with the axial anomer of glucose. However, the unfavorable effect of this eclipsing would be counteracted, in some measure, by the release of strain arising from separation of the hydroxyl groups of $\mathrm{C} 1$ and $\mathrm{C} 3$, and hence the change in conformation, as with the manno and talo configurations, would require less energy than that required in the glucose series. The rate of oxidation for $\beta$-D-lyxose was found to be low in comparison with the rates for the configurationally related aldoses, $\beta$-D-mannose and $\mathrm{D}$-glycero- $\beta$ - $\mathrm{L}-$ manno-heptose, but it is close to the rates for $\beta$-Dgulose and D-glycero- $\beta$-D-gulo-heptose. The relatively low rate of oxidation for $\beta$-D-lyxose, and the high rate for $\alpha$-D-lyxose, arises at least in part from a fast 
anomerization reaction. (See p. 147 of [3].) The unusually small ratio for the rates could be explained equally well by a non-chair conformation for one or both of the anomers. It is noteworthy that Reeves predicted for $\beta$-D-lyxose a mixture of the chair conformations or, possibly, a conformation in the boatskew cycle.

The aldoses of group 3 behave somewhat differently from those of groups 1 and 2 . The freshly dissolved, crystalline aldoses are oxidized at intermediate rates, and the oxidations of the aldoses present in equilibrium mixtures do not show abrupt changes in rate. Small changes in the rates during the oxidations indicate that the equilibrium solutions of both D-allose and D-ribose contain a small proportion (possibly 10\%) of a modification oxidized more rapidly than the rest of the aldose. Both aldoses exhibit complex mutarotation reactions, presumably involving both pyranose and furanose modifications. D-Altrose, in an equilibrium solution, was found to be oxidized at essentially the same rate as the freshly dissolved aldose, and a carefully purified solution of D-idose was found to be oxidized at a uniform rate, about six times that of $\alpha$-D-glucose. Neither D-altrose nor D-idose gave evidence for a substantial proportion of a second modification in the equilibrium mixture. ${ }^{7} \quad$ D-glycero-D-ido-Heptopyranose is the only known crystalline aldose having the $i d o$ configuration. When the aldose was first crystallized [31], an unusual conformation was suspected (see p. 530 of [6]); this is now considered to be $\mathrm{CE}$, the conformation in which the $\alpha$ anomer has an equatorial C1-hydroxyl group. Oxidation of freshly dissolved crystalline D-glycero-D-ido-heptose takes place about 11 times as rapidly as that of $\alpha$-D-glucose. Oxidation of an equilibrium solution of D-glycero-D-ido-heptose gave evidence for the presence of a small proportion of a slowly oxidizable modification; the character of this modification remains to be determined.

Interpretation of the oxidation of D-glycero-D-idoheptose and certain other sugars is hampered by lack of knowledge of their ring structures and anomeric configurations. When both anomers are known, the anomeric configuration of pyranoses can be assigned on the basis that the more dextrorotatory (or less levorotatory) anomer of an alpha-beta pair has the D-glycero configuration at $\mathrm{C} 1$. When only one crystalline anomer is known and the mutarotation reaction is complex, the configuration cannot be assigned from the optical rotation; this is the case with crystalline D-ribose, D-allose, D-altrose, and D-glycero-D-ido-heptose. In re-examining the classification of these aldoses, advantage was taken of the observation of Haworth and Hirst [32] that the anomer that preponderates in an equilibrium solution of a pyranose has trans hydroxyl groups at $\mathrm{C} 1$ and C2. This principle is applicable for tentative assignment of configuration, because (as shown in table 2) all aldoses of known configuration conform thereto. Thus, if the aldoses are present largely in the pyranoid form, we can conclude that the preponderating

7 The bromine-oxidation measurements for D-allose, D-altrose, and D-idose were conducted by R. Schaffer and B. Y. Foley.
TABLE 2. Equilibrium proportions of the anomeric aldopyranoses ${ }^{\mathrm{a}}$

\begin{tabular}{|c|c|c|c|c|}
\hline \multirow[b]{2}{*}{ Aldose } & \multicolumn{4}{|c|}{ Composition of the equilibrium solution } \\
\hline & \multicolumn{2}{|c|}{$\begin{array}{l}\text { Estimated, from } \\
\text { bromine oxidation }\end{array}$} & \multicolumn{2}{|c|}{$\begin{array}{l}\text { Calculated, from } \\
\text { optical rotation, } \\
\text { assuming only two } \\
\text { constituents }\end{array}$} \\
\hline $\begin{array}{l}\text { L-Arabinose } \\
\text { D-Lyxose } \\
\text { D-Ribose }\end{array}$ & $\begin{array}{c}\text { cis } \\
\text { anomer b } \\
32.4 \\
20.3\end{array}$ & \begin{tabular}{|c|} 
trans \\
anomer b \\
67.6 \\
79.7
\end{tabular} & $\begin{array}{c}\text { cis } \\
\text { anomer b } \\
26.5 \\
24.0\end{array}$ & $\begin{array}{r}\text { trans } \\
\text { anomer b } \\
73.5 \\
76.0\end{array}$ \\
\hline $\begin{array}{l}\text { D-X ylose } \\
\text { D-Allose }\end{array}$ & 32.1 & 67.9 & 34.8 & 65.2 \\
\hline $\begin{array}{l}\text { D-Altrose }{ }^{\text {C }} \\
\text { D-Galactose } \\
\text { D-Glucose } \\
\text { D-Gulose (calcium chloride) } \\
\text { D-Idose }\end{array}$ & $\begin{array}{l}31.4 \\
37.4 \\
18.5\end{array}$ & $\begin{array}{l}68.6 \\
62.6 \\
81.5\end{array}$ & $\begin{array}{l}29.6 \\
36.2 \\
-\end{array}$ & $\begin{array}{r}70.4 \\
63.8 \\
-\end{array}$ \\
\hline $\begin{array}{l}\text { D-Mannose. } \\
\text { D-Talose. }\end{array}$ & $\begin{array}{l}31.1 \\
44.1\end{array}$ & $\begin{array}{l}68.9 \\
55.9\end{array}$ & 31.2 & 68.8 \\
\hline $\begin{array}{l}\text { L-Rhamnose } \\
\mathrm{D}-\text { glycero-D-galacto-Heptose }\end{array}$ & 31.0 & 69.0 & 26.9 & 73.1 \\
\hline $\begin{array}{l}\text { D-glycero-D-galacto-Heptose } \\
\text { D-glycero-L-galacto-Heptose }\end{array}$ & $\begin{array}{l}32.8 \\
37.0\end{array}$ & $\begin{array}{l}67.2 \\
63.0\end{array}$ & 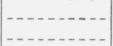 & (n) \\
\hline $\begin{array}{l}\text { D-glycero-L-gluco-Heptose. } \\
\text { D-glycero-D-gulo-Heptose }\end{array}$ & 37.2 & 62.8 & (n) & - \\
\hline $\begin{array}{l}\text { D-glycero-D-gulo-Heptose } \\
\text { D-glycero-D-ido-Heptose }\end{array}$ & 11.8 & 88.2 & & \\
\hline D-glycero-L-manno-Heptose............. & 20.6 & 79.4 & 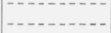 & $-\cdots$ \\
\hline $\begin{array}{l}\text { D-glycero-D-talo-Heptose } \mathrm{d} \\
\text { D-glycero-L-talo-Heptose } \mathrm{d}\end{array}$ & $\begin{array}{l}38 \\
33\end{array}$ & $\begin{array}{l}62 \\
67\end{array}$ & (n) & -............. \\
\hline $\begin{array}{l}\text { D-glycero-L-talo-Heptose a } \\
\text { Lactose }\end{array}$ & 37.5 & 62.5 & 36.8 & 63.2 \\
\hline Maltose_............. & 37. 7 & 62.3 & 36.0 & 64.0 \\
\hline
\end{tabular}

a Except where indicated, these data were compiled from the work of Isbell and Pigman given in chapter XXIX of reference [5].

$\mathrm{b}$ In anomers designated cis, the hydroxyl groups at $\mathrm{C} 1$ and $\mathrm{C} 2$ lie on the same

$\mathrm{b}$ In anomers designated cis, the hydroxyl groups at $\mathrm{C} 1$ and $\mathrm{C} 2$ lie on the same
side of the ring: in anomers designated trans, they lie on opposite sides of the ring.

Knowledge of configuration and properties is insufficient for classification of the anomers.

d Values calculated from data reported in J. Research NBS 20, 97 (1938) RP1069.

constituents in the equilibrium solutions of the above aldoses are respectively, $\beta$-D-ribopyranose, $\beta$-D-allopyranose, $\alpha$-D-altropyranose, and D-glycero$\alpha$-D-ido-heptopyranose. Except for the last-named aldose, these assignments agree with the structures previously accepted. Classification of crystalline D-glycero-D-ido-heptose as an $\alpha$-D-pyranose in the CE conformation constitutes an important change, and all work on this compound that has been based on the assumption that it was the $\beta$ anomer will need to be revised. On the basis of the trans anomer rule, it is postulated that the $\alpha$ pyranose modification of D-idose, also, preponderates in aqueous solutions.

Inasmuch as the rates of oxidation depend on the free energy of the reactants in the ground state as well as in the transition state, it is of interest to compare the free energies of the aldoses in aqueous solution. The difference in free energy for a pair of anomeric aldoses in solution can be estimated from the proportions of the anomers at equilibrium. The rates of oxidation clearly show, for aldoses in the gluco series, that the anomers having equatorial C1-hydroxyl groups preponderate in the equilibrium mixture. Thus, the free energy of $\beta$-D-glucose in the oxidation mixture is less than that of $\alpha$-D-glucose. With aldoses in the manno series, the anomer with the axial C1-hydroxyl group preponderates at equilibrium, and $\alpha$-D-mannose in solution must have a lower free-energy than $\beta$-D-mannose. Quantitative evaluation of the free energies for the various sugars in aqueous solutions would be desirable but it is not feasible at present for lack of precise knowledge concerning the composition of the equilibrium mixtures. 


\section{Comparison of the Oxidation of Aldoses With the Oxidation of Cyclohexanols}

In comparison with the aldopyranoses, cyclohexanol and its derivatives are oxidized slowly, and the relative rates of oxidation of the axial and equatorial isomers by either chromic acid [33] or bromine [22] are reversed. According to Barker, Overend, and Rees [22], the difference in the rates of oxidation for the axial and equatorial isomers of tert-butylcyclohexanol is far less than the difference in rates for $\alpha$ and $\beta$-D-glucose. The authors propose the mechanism depicted in figure 4. Simultaneous removal of the two hydrogen atoms in the transition state is postulated to account for a lack of sensitivity of the rate to a change in acidity.

The oxidation of cyclohexanols differs from that of aldoses in several important respects. With aldoses, but not with cyclohexanols, it is postulated by the author that resonance involving the ring-oxygen atom aids in the release of the C1-hydrogen atom. This hypothesis may account for the more rapid oxidation of aldoses. In the oxidation of cyclohexanols, the C1-oxygen atom does not lie in the same plane as $\mathrm{C} 1, \mathrm{C} 2, \mathrm{C} 5$, and $\mathrm{C} 6$, because the $\mathrm{C} 1-$ C6 bond (corresponding to the $\mathrm{C} 1-\mathrm{O}$ bond of the pyranose ring) has no double-bond character, and there is consequently no resonance stabilization of such a planar conformation. For this reason, the C1-hydroxyl group of the cyclohexanols can approach the transition state from either the axial or the equatorial position without a change in the conformation of the molecule. This accounts for the lack of a large difference in the rates of reaction for the axial and equatorial isomers. Because of the intramolecular repulsive forces, a cyclohexanol having an axial hydroxyl group would have a higher free-energy in both ground and transition states than a similar one having an equatorial hydroxyl group, and less energy would be required for the axial isomer to reach the transition state than for the equatorial isomer. However, the difference in the free energies in the transition states would be less than that in the ground states because of modification of the repulsive forces by bromine. Consequently, less energy would be required for the axial isomer to reach the transition state than for the equatorial isomer, and, therefore, the rate of reaction should be higher for the axial isomer, as it, in fact, is. The relationship is opposite to that for the anomeric aldoses.

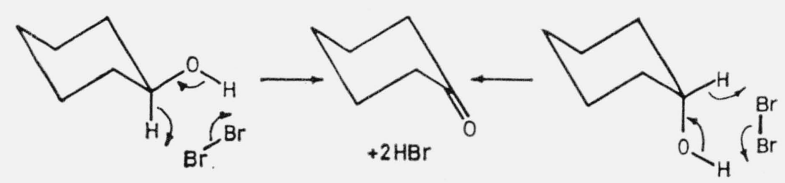

Figure 4. Oxidation of axial and equatorial isomers of tert-butylcyclohexanol according to Barker, Overend, and Rees [22].)

\section{Summary}

Early studies of the oxidation of aldoses by the author revealed that the ring forms of the aldoses are converted to lactones without cleavage of the ring, that free bromine is the oxidant, and that there are wide differences in the rates of oxidation for the different aldoses and for the $\alpha$ and $\beta$ modifications of a single aldose. The hypothesis was advanced that the rates of reaction of the anomers depend on the angular position of the C1-hydroxyl group with respect to the plane of the ring. So far as known, this was the first attempt in any field of chemistry to correlate reaction rate and conformation. Recent reappraisal of the results by means of present concepts of reaction-rate theory and of ring conformation leads to the hypothesis that the differences in the rates of oxidation for the $\alpha$ and $\beta$ anomers arise from variation in the free energy involved in conversion of the reactants in their ground states to transition states in which the oxygen atom of the C1-hydroxyl group lies in the plane formed by the ring-oxygen atom, C1, C2, and C5. Presumably, this conformation results from stabilization by resonance.

In the oxidation of derivatives of cyclohexanol, the oxygen atom, in the transition state, does not need to lie in the same plane as C1, C2, C5, and C6 because, in the absence of a ring-oxygen atom, there is no resonance stabilization of such a conformation. This hypothesis may account for the lower rates of oxidation of the cyclohexanols, for lack of wide differences in the rates of oxidation of the axial and equatorial isomers, and for the fact that the relative rates of oxidation of the isomers are reversed from those of the axial and equatorial anomers of the aldopyranoses.

The author expresses his appreciation to $\mathrm{R}$. U. Lemieux of the University of Toronto, and to $\mathrm{H}$. Eyring of the University of Utah for highly valued counsel. He thanks R. S. Tipson and H. L. Frush for assistance in preparing the manuscript, and $\mathrm{R}$. Schaffer and B. Y. Foley for measurement of the rates of oxidation for D-allose, D-altrose, and D-idose.

\section{References}

[1] H. S. Isbell and C. S. Hudson, BS J. Research 8, 327 (1932) RP418.

[2] H. S. Isbell, BS J. Research 8, 615 (1932) RP441.

[3] H. S. Isbell and W. W. Pigman, J. Research NBS 18, 141 (1937) RP969.

[4] H. S. Isbell and W. W. Pigman, BS J. Research 10, 337 (1933) RP534.

[5] F. J. Bates and Associates, Polarimetry, Saccharimetry, and the Sugars, NBS Circ. 440 (1942).

[6] H. S. Isbell, J. Research NBS 18, 505 (1937) RP990.

[7] R. E. Reeves, J. Am. Chem. Soc. $\boldsymbol{\gamma 1}, 215,1737,2116$ (1949); 72, 1499 (1950).

[8] R. E. Reeves, Advances in Carbohydrate Chem. 6, 107 (1951); Ann. Rev. Biochem. 2\%, 15 (1958).

[9] O. Hassel and B. Ottar, Acta Chem. Scand. 1,929 (1947). 
[10] C. W. Beckett, K. S. Pitzer, and R. Spitzer, J. Am. Chem. Soc. 69, 2488 (1947).

[11] R. Bentley, Nature 176, 870 (1955).

[12] H. H. Bunzel and A. P. Mathews, J. Am. Chem. Soc. 31, 464 (1909).

[13] B. Perlmutter-Hayman and A. Persky, J. Am. Chem. Soc. 82, $276(1960)$.

[14] I. R. L. Barker, W. G. Overend, and C. W. Rees, Chem. \& Ind. (London), 1960, 1297, 1298.

[15] R. Bentley, J. Am. Chem. Soc. 79, 1720 (1957).

[16] F. Shafizadeh, Advances in Carbohydrate Chem. 13, 9 (1958)

[17] N. N. Lichten and M. H. Saxe, J. Am. Chem. Soc. 7\%, 1875 (1955).

[18] F. Friedberg and L. Kaplan, Abstracts Papers Am. Chem. Soc. 131, 86 o (1957).

[19] L. Kaplan, J. Am. Chem. Soc. 76, 4645 (1954); 80, 2639 (1958).

[20] H. S. Isbell, Abstracts Papers Am. Chem. Soc. 132, $1 \mathrm{D}$ (1957).

[21] C. G. Swain, R. A. Wiles, and R. F. W. Bader, J. Am. Chem. Soc. 83, 1945 (1961).

[22] I. R. L. Barker, W. G. Overend, and C. W. Rees, Chem. \& Ind. (London) 1961, 558.
[23] H. S. Isbell, Chem. \& Ind. (London) 1961, 593.

[24] H. Eyring, Chem. Revs. 1\%, 65 (1935).

[25] N.-J. Chü, Conformational analysis of the equilibria and rates for the anomerization of acetylated aldopyranoses, Ph.D. Thesis (University of Ottawa, Canada, Feb. 1959).

[26] M. S. Newman, Steric Effects in Organic Chemistry, p. 152 (John Wiley \& Sons, Inc., New York, 1956).

[27] E. S. Gould, Mechanism and Structure in Organic Chemistry, p. 178 (Henry Holt and Company, New York, 1959).

[28] H. S. Isbell and R. S. Tipson, Science 130, 793 (1959); J. Research NBS 64A, 171 (1960).

[29] H. S. Isbell and H. L. Frush, J. Research NBS 24, 125 (1940) RP1274.

[30] H. S. Isbell, J. Research NBS 5\%, 171 (1956) RP2707.

[31] H. S. Isbell, J. Am. Chem. Soc. 56, 2789 (1934).

[32] W. N. Haworth and E. L. Hirst, J. Chem. Soc. 1928, 1226.

[33] S. Winstein and N. J. Holness, J. Am. Chem. Soc. 7y, $5562(1955)$.

(Paper 66A3-157) 